\title{
Correction to: Proper locally spherical hypertopes of hyperbolic type
}

\author{
Antonio Montero $^{1}$ (D) Asia Ivić Weiss $^{2}$ (D) \\ Published online: 15 December 2021 \\ (c) Springer Science+Business Media, LLC, part of Springer Nature 2021
}

Keywords Regularity $\cdot$ Thin geometries $\cdot$ Hypermaps $\cdot$ Hypertopes $\cdot$ Abstract polytopes

Mathematics Subject Classification Primary: 52B15 - 51E24 - Secondary: 51G05

\section{Correction to: Journal of Algebraic Combinatorics https://doi.org/10.1007/s10801-021-01054-6}

The original version of this article, published online on 25 October 2021 (https:// doi.org/10.1007/s10801-021-01054-6), has some imprecisions that are described and fixed in this document.

- There is a missing reference in page 7 :

In [29][?] the graphs were used to build [...]

The correct reference is [1]

- Lemma 2 in page 8 is poorly formatted. The correct text should be as follows.

Lemma 2 Let $\mathcal{X}$ be apropern-edge-coloured graph with colour-set $I=\{0, \ldots, n-1\}$. For $i \in I$, let $\rho_{i}$ denote the permutation of the vertices of $\mathcal{X}$ induced by the matching $M_{i}$. Let $p_{1}, \ldots p_{r}, q_{1}, \ldots, q_{s} \in \mathbb{N}$ such that all the $\{i, j\}$-components of $\mathcal{X}$ are

The original article can be found online at https://doi.org/10.1007/s10801-021-01054-6.

$凶$ Antonio Montero

amontero@im.unam.mx

Asia Ivić Weiss

weiss@yorku.ca

1 Institute of Mathematics, National Autonomous University of Mexico (IM UNAM), 04510 Mexico City, Mexico

2 Department of Mathematics and Statistics, York University, Toronto, ON M3J 1P3, Canada 
alternating paths with $p_{k}$ vertices or alternating cycles with $2 q_{l}$ vertices for some $1 \leq k \leq r$ and $1 \leq l \leq s$. Then, the period of $\rho_{i} \rho_{j}$ is $\operatorname{lcm}\left(p_{1}, \ldots p_{r}, q_{1}, \ldots, q_{s}\right)$.

\section{Reference}

1. Pellicer, D.: Extensions of dually bipartite regular polytopes. Discrete Math. 310(12), 1702-1707 (2010). https://doi.org/10.1016/j.disc.2009.11.023. (ISSN: 0012-365X)

Publisher's Note Springer Nature remains neutral with regard to jurisdictional claims in published maps and institutional affiliations. 